\title{
Obesity Associated with Low Lean Mass and Low Bone Density Has Higher Impact on General Health in Middle-Aged and Older Adults
}

\author{
Natasha A. G. de França $\left(\mathbb{D},{ }^{1,2}\right.$ Barbara S. E. Peters $\left(\mathbb{D},{ }^{1}\right.$ Elizabete A. dos Santos ${ }^{(D)}{ }^{1}$ \\ Marcela M. S. Lima $\mathbb{D}^{1},{ }^{1}$ Regina M. Fisberg $\mathbb{D}^{1},{ }^{1}$ and Ligia Araújo Martini $\mathbb{D}^{1}$ \\ ${ }^{1}$ Department of Nutrition, School of Public Health, University of Sao Paulo (USP), São Paulo, São Paulo, Brazil \\ ${ }^{2}$ Centro Universitario de Rio Preto (Unirp), São José do Rio Preto, São Paulo, Brazil \\ Correspondence should be addressed to Natasha A. G. de França; natasha.agf@gmail.com
}

Received 6 July 2020; Revised 14 November 2020; Accepted 20 November 2020; Published 3 December 2020

Academic Editor: Claire Stocker

Copyright (c) 2020 Natasha A. G. de França et al. This is an open access article distributed under the Creative Commons Attribution License, which permits unrestricted use, distribution, and reproduction in any medium, provided the original work is properly cited.

It is believed that the phenomenon of simultaneous changes in body composition could have a higher negative impact on general health. Thus, we aimed to investigate the prevalence of concomitant body composition disturbances and evaluate the association with dietary intake, sedentary behaviour, muscle strength, and performance. This is a cross-sectional study with 218 communitydwelling adults, aged 63 (59-69) years, both sexes (52\% female) recruited from the Health Survey of the City of São Paulo. Assessments include appendicular lean mass (LM), fat mass and bone mineral density (BMD) by DXA, grip strength, time spent sitting, and dietary intake. Subjects were clustered into 8 groups: (1) normal, (2) osteopenia (OP), (3) low LM, (4) obesity, (5) OP + low LM, (6) obesity + OP, (7) obesity + low LM, and (8) obesity + OP + low LM. Statistical analyses include ANCOVA, the chi-square test, and linear regression models. 52 (23\%) individuals presented obesity associated with another body composition change, with $14(6 \%)$ having the combination of the 3 conditions (obesity + OP + low LM). All groups with obesity showed lower protein intake $(p \leq 0.001)$; however, those with obesity or obesity + low LM spent more time in a sitting position $(p=0.002)$, and the group with obesity $+\mathrm{OP}+$ low LM had the lowest grip strength. The combination of obesity with low LM and OP presented the aggravating factor of being associated with lower grip strength. In a context of demographic and nutrition transition, the findings represent a demand for longitudinal investigations.

\section{Introduction}

Simultaneous osteopenia/osteoporosis, low lean mass/sarcopenia, and obesity have been defined as a syndrome, osteosarcopenic obesity [1]. The diagnosis depends on the diagnosis of each component, which makes it harder to establish its prevalence. The prevalence and impact of osteosarcopenia, which describes low lean mass associated with reduced bone mineral density (BMD), are well described in current literature [2-6]. Such interest emerged from observations in clinical practice where a higher prevalence of osteopenia has been observed among older people with sarcopenia and vice versa. The skeletal muscle and its strength, as well as BMD, follow a similar path, showing an imbalance in the bone remodelling and protein synthesis/degradation ratio with aging, mainly from 50 years of age. Compared to young muscle and bone, it is estimated that there is a $30 \%$ reduction in these tissues and has been estimated to occur when patients are aged 75 years [7].

Less commonly described is the extent to which condition obesity and reduced lean mass (sarcopenic obesity) and obesity with osteopenia as higher body mass index (BMI) usually reflect higher fat but also higher lean mass [8], given its recognition as a protective factor for lower BMD. However, assessing a population of Asian adults, Kim et al. (2014) [9] observed a positive association between BMD and muscle mass, while noting that body fat was only positively related to BMD among eutrophic subjects (BMI $<25 \mathrm{~kg} / \mathrm{m}^{2}$ ). The findings of Kim et al. suggest that the protective effect of fat does not extend to obesity. In this way, a state of obesity with sarcopenia may have a higher negative impact on BMD, thereby increasing the risk of osteoporosis and fractures [10]. 
Obesity, mainly related to aging, is accompanied by a redistribution of body fat, with increased deposits in the abdominal area and infiltration into musculoskeletal tissue. Fat redistribution has an impact on bone and muscle quality, which may lead to a higher risk of falls, fractures, and frailty $[11,12]$. In addition, the reduction in muscle mass and its performance is associated with lower physical activity, leading to a decrease in energy expenditure, as well as in the stimulus for protein synthesis and bone remodelling. Thus, the co-occurrence of these conditions represents a vicious cycle, contributing to the maintenance of obesity, reduced lean mass, and osteopenia [13].

Beyond aging physiology, both sedentary lifestyle and unbalanced diet are considered important factors that negatively impact body composition. It has been suggested that low intake of protein, unsaturated fat (mainly omega 3), calcium, and vitamin $D$ may mediate obesity, sarcopenia, and osteopenia. Such nutrients have been considered diet components that are potentially involved in the development of the concomitant disturbances of body composition [14, 15]. Furthermore, adipose tissue, skeletal muscle, and bone all have endocrine functions, synthesizing hormones and releasing cytokines that can also have a paracrine performance [1].

Each change in body composition can compromise the working capacity of adults, as well as the frailty status and risk of death among older persons. Thus, the association of such disturbances is believed to have the potential to increase the impact on quality of life and general health. However, exploring concomitant changes in body composition is a relatively recent development in the literature. It is necessary to evaluate whether all possible body changes combinations are significantly prevalent among different populations and in different clinical situations. Beyond prevalence, it is also essential to define the real impact and the characteristics (physiological, metabolic, socioeconomic, and lifestyle) to better screen individuals at higher risk for simultaneous body composition changes and to strategize more effective public health policies for prevention and treatment.

The objective of this study was to investigate the prevalence of concomitant disturbances of body composition (obesity, low lean mass, and osteopenia) in a sample of community-dwelling adults, from 50 years of age, and evaluate the association of such disturbances with dietary intake, sedentary behaviour, muscle strength, performance, and sociodemographic characters. The hypothesis was that an increased number of simultaneous body composition changes are associated with sedentary lifestyle and unbalanced diet (especially in the proportion of macronutrients), strength, and performance.

\section{Materials and Methods}

2.1. Design and Study Population. In this cross-sectional study, participants were recruited from the "Health Survey of the City of São Paulo" (ISA-Capital 2015) [16] and "2015 Health Survey of São Paulo with Focus in Nutrition Study" (2015 ISA-Nutrition) [17]. Briefly, ISA-Capital 2015 is a cross-sectional population-based survey composed of a probabilistic sample of residents of São Paulo City, Brazil, which aimed to evaluate health status, lifestyle, and use of health services. A subsample of the ISA-Capital 2015 was drawn to compose the 2015 ISA-Nutrition, a household cross-sectional, population-based survey designed to evaluate the association of modifiable lifestyle-related factors with biochemical and genetic markers, and the environmental factors related to the development of cardiometabolic disease [17].

For this study, we recruited all participants over 50 years, of both sexes, who had completed all the assessments of both ISA-Capital 2015 and 2015 ISA-Nutrition. It was recommended that sample size approximate 240 participants, considering a study power $(\beta-1)$ of $80 \%$ using the $\mathrm{G} *$ Power software [18]. Ineligibility criteria included current or recent cancer (self-reported), corticosteroids, antineoplastic agents and hormonal inhibitors, important mobility or neurological disorders (e.g., Parkinsonism), and current or recent pregnancy. All criteria for exclusion are presented in Figure 1. All procedures performed in the study were in accordance with the ethical standards of the Ethics Committee on Research of the Public Health School, University of São Paulo (44552815.0.0000.5421) and with the 1964 Helsinki declaration and its later amendments, and written informed consent was obtained from all the participants.

2.2. Body Composition Assessment and Clustering. Weight (kg) and height (m) were obtained, after which, BMI was calculated. Lean mass, fat mass, and BMD were assessed by DXA (iDXA Advance; GE Healthcare, Madison, WI, EUA). Classification is described as follows.

2.3. Lean Mass. A DXA scan of the total body obtained appendicular lean mass, which was adjusted for BMI and classified according to the recommendations of the Foundation for the National Institutes of Health (FNIH) [19]. Values $<0.789$ for men and $<0.512$ for women were set as low lean mass.

2.4. Fat Mass. Obesity was also defined using DXA parameters. We applied the fat mass index (FMI) [20], in which total body fat $(\mathrm{kg})$ is adjusted by height squared $\left(\mathrm{m}^{2}\right)$. Obesity was determined when FMI was $>9 \mathrm{~kg} / \mathrm{m}^{2}$ and $>13 \mathrm{~kg} / \mathrm{m}^{2}$ in men and women, respectively. Visceral adipose tissue (VAT, kg) was also assessed (continuous only).

2.5. Bone. DXA scans were performed on the femoral neck (FN) and lumbar spine (LS; L1-L4). Osteopenia/osteoporosis was defined according to the WHO standard criteria of having a $T$-score of less than $-1.0 \mathrm{SD}$ in at least one of these sites [21].

Subjects were clustered into groups, according to the presence/absence of body composition disturbances, resulting in 8 groups: (1) normal (without body composition disturbances), (2) osteopenia/osteoporosis (OP), (3) low lean mass (low LM), (4) obesity, (5) osteopenia/osteoporosis plus low lean mass (OP + low LM), (6) obesity plus 


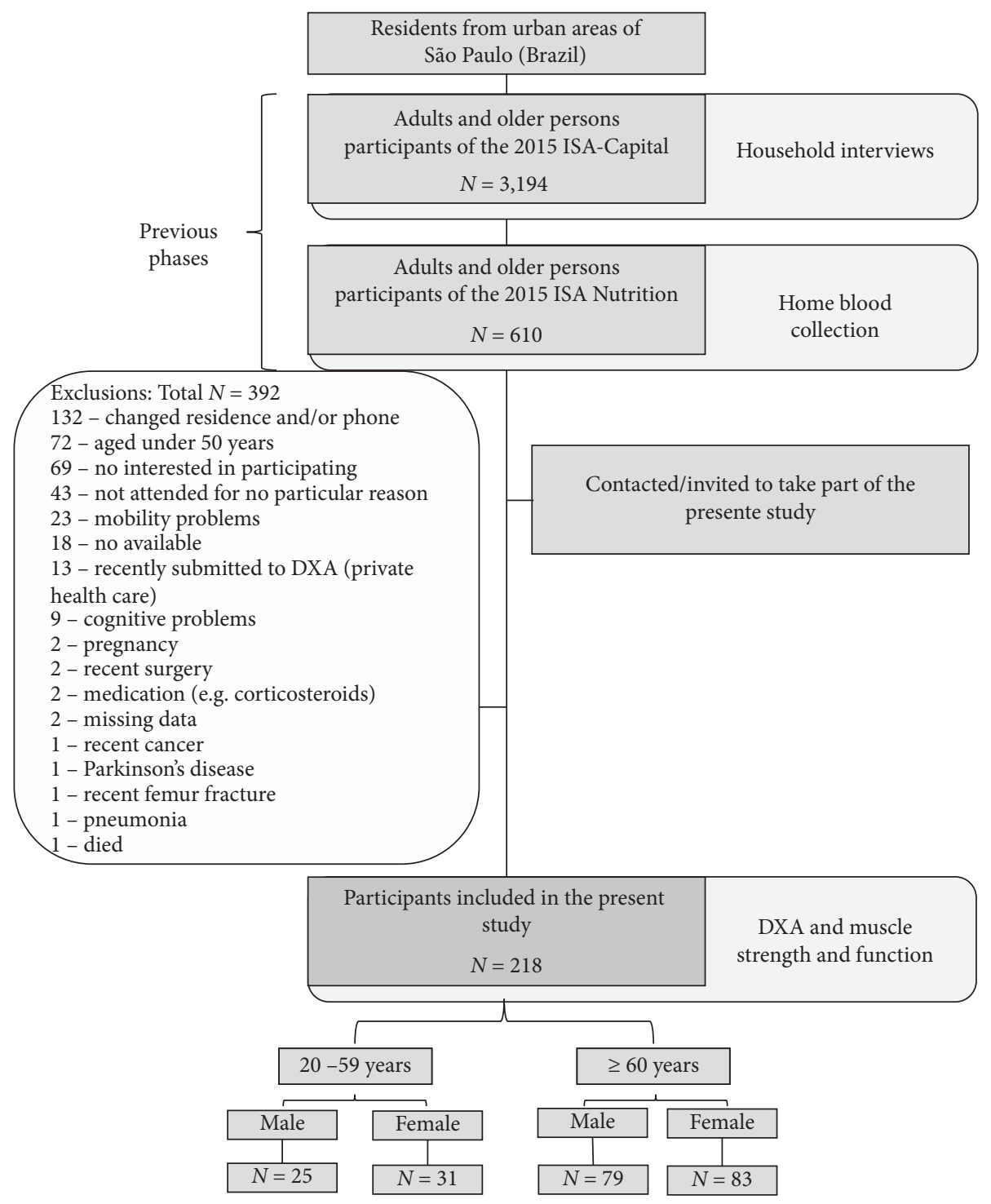

FIgURE 1: Study flowchart.

osteopenia/osteoporosis (obesity + OP), (7) obesity plus low lean mass (obesity + low LM), and (8) obesity associated with osteopenia/osteoporosis and low lean mass (obesity + OP + low LM).

2.6. Physical Performance, Strength, and Sedentary Behavior. Grip strength was tested using a hand dynamometer (Jamar ${ }^{\circledR}$, Jackson, MI, EUA). The best effort from three attempts with both hands (with $60 \mathrm{~s}$ of rest between each attempt) was defined as the grip strength. We recorded gait speed from participants walking $4 \mathrm{~m}$ at their usual pace. The average of two takes was divided by the distance $(4 \mathrm{~m})$ and considered for analysis.

Sedentary behavior was obtained by employing the long form of the International Physical Activity Questionnaire (IPAQ), which includes a question about time spent sitting at work, at home, while doing course work, and during leisure time (min/week). Time spent in motorized transportation is not included in this domain.
2.7. Food Intake. Dietary intake assessment was collected by employing two dietary recall questionnaires performed on nonconsecutive days. The information was imputed into the Nutrition Data System for Research (NDSR) software (version 2014; Nutrition Coordinating Center, University of Minnesota, Minneapolis, MN, USA). Usual dietary intake was estimated using statistical modeling techniques incorporated into the Multiple Source Method program [22]. For more information about dietary assessment, see Fisberg et al. (2018) [17]. For assessing nutrient intake, we considered the Dietary Reference Intakes determined by the Institute of Medicine (DRI, IOM) [23, 24].

2.8. Sociodemographic and General Covariates. Additional variables included smoking habit (yes/no) and habitual alcohol consumption (yes/no), race (white/nonwhite), educational level $(\leq 9,10-12$, or $\geq 13$ years of study), marital status (married/not married), income per capita (according to the Brazilian minimal wage in 2015; $\leq$ US\$217.32, 
US\$217.32-US\$651.96, and > US\$651.96 per month), and medications in use (type and number $0,1-4$, and $\geq 5$ ). All information was provided from the standardized protocols applied during the ISA-Capital 2015.

2.9. Statistical Analysis. Analyses were performed using Stata Statistical Software, version 13.1 (StataCorp LP, College Station, TX, USA), and the two-tailed $p$ value of $<0.05$ was considered significant. Continuous variables were tested for normality with the Shapiro-Wilk test, and those not normally distributed $(p<0.05)$ were standardized to score-z. Descriptive results are presented as median (interquartile ranges) and frequencies (\%), as appropriated.

Aiming to better explore the differences in continuous variables among the 8 groups, we performed one-way ANOVA, adjusted for age and sex (ANCOVA), followed by the Tukey-Kramer post hoc test. For analysis of the categorical variables, it was necessary to regroup participants into fewer groups before performing the chi-squared test, since the original grouping resulted in some categories with few cases, making the test unfeasible. We opted to regroup the individuals as follows: (1) normal, (2) low LM and/or OP, (3) obesity, and (4) obesity + OP and/or low LM. Considering muscle strength and performance as the main outcomes, the impact of each body composition component (body fat, lean mass, and BMD) on such variables was tested via linear regression models. The models were performed for male and female separately. All models were first performed unadjusted (Model 1), then controlled for the other body composition variables (Model 2), and for Model 2 plus protein intake $(\mathrm{g} / \mathrm{kg})$, time spent sitting (min/week), and age (years) (Model 3). Multicollinearity and heteroskedasticity were checked by inspection of the variance inflation factor (VIF) and Breusch-Pagan test, respectively.

\section{Results and Discussion}

3.1. Results. Of the 610 adults and older participants of 2015 ISA-Nutrition, 132 were not reached due to changes in phone number or address, 72 were younger than 50 years, 143 refused to participate for different reasons, and 45 were excluded, according to the exclusion criteria (Figure 1). Thus, the final sample consisted of 218 subjects, aged 63 (59-69) years, with most of them older adults $(74 \%)$, women $(52 \%)$, and white $(62 \%)$. Among the whole sample, the average time spent in sedentary activities was 1,260 (735-2,100) min per week, and dietary assessment showed that at least $25 \%$ of the participants had very low protein intake $(<0.7 \mathrm{~g} / \mathrm{kg})$ and high-saturated fat intake ( $>11 \%$ of energy intake). Median calcium and vitamin $D$ intakes were also below the recommendations: $535(382-655) \mathrm{mg} /$ day and $1.5(0.96-2.2)$ $\mu \mathrm{g} /$ day, respectively. Most subjects were married $(N=123$; $57 \%)$, had low educational level $(N=115 ; 53 \%)$, a per capita income of up to 1 minimum wage $(N=110 ; 51 \%)$, did not smoke $(N=132 ; 61 \%)$, or usually drink alcohol $(N=164$; $75 \%$ ). The prevalence of polypharmacy ( $\geq 5 \mathrm{drugs}$ ) was $21 \%$ $(N=46)$ (data not shown). Body composition assessment and diagnoses resulted in different prevalence across conditions (Figure 2).

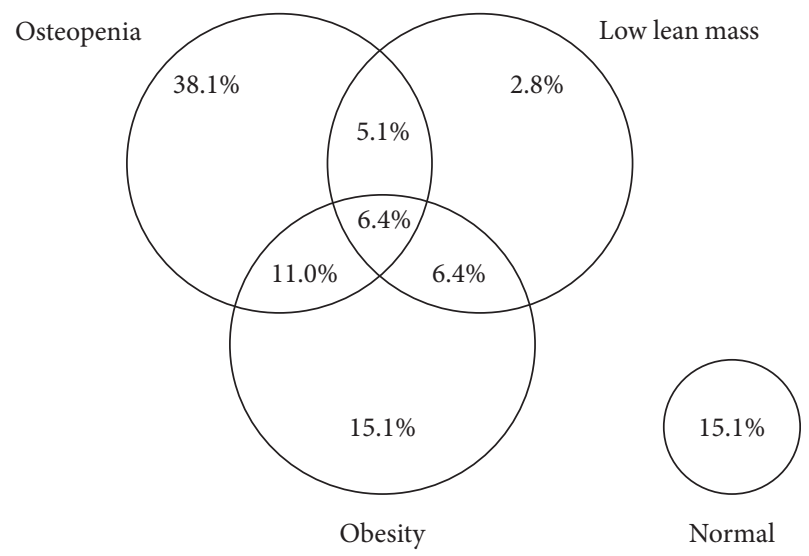

FIGURE 2: Venn diagram of overlap of the frequencies of osteopenia, low lean mass, and obesity in the study population. "Normal" circle represents those without disturbances in body components.

A comparison revealed that VAT, strength, time spent sitting, and protein intake differed across all 8 groups (Table 1). The post hoc test showed that those with obesity (exclusive or associated with another body composition change) had approximately twofold more VAT than the groups with proper fat mass ( $p \leq 0.001$; Table 1$)$. Regarding muscle strength, the groups OP $(26.6 \pm 9.3 \mathrm{~kg})$ and obesity + OP + low LM $(23.5 \pm 6.9 \mathrm{~kg})$ were weaker than normal $(33 \pm 9.7 \mathrm{~kg})$ and obesity $(33 \pm 9.9 \mathrm{~kg})$ groups $(p \leq 0.001)$. Lifestyle habits showed that those with obesity $(2,184 \pm 1,680 \mathrm{~min} /$ week $)$ or obesity + low LM $(2,752 \pm 1,770 \mathrm{~min} /$ week $)$ spent longer time in sitting position than those normal for body composition $(1,314 \pm 866 \mathrm{~min} /$ week $)$ and OP $(1,350 \pm 1,055 \mathrm{~min} /$ week $)$ $(p=0.002)$. Additionally, all groups with obesity (obesity; obesity + OP; obesity + low LM; and obesity + OP + low LM) had the lowest protein intake by bodyweight $(p \leq 0.001)$ (Table 1).

For categorical variables, we performed a chi-squared test with participants allocated in 4 groups: (1) normal, (2) low LM and/or OP, (3) obesity, and (4) obesity + OP and/or low LM (Table 2). Analyzing residuals of the test showed that being female was positively associated with having $\mathrm{OP}$ and/ or low LM, while being male was inversely associated with having such conditions $(p=0.054)$. We also observed that a better income (>US\$ 651.96/month) was associated with being obese $(p=0.021)$.

Table 3 shows the results for linear regression models designed to test which body composition components can predict deviations in strength (no significant model was observed for gait speed). In the male model, in addition to both LM and total body fat having a significant impact on strength, only LM retained a positive association with strength after controlling for protein intake, time spent sitting, and age. Thus, among males, each $10 \%$ increase in LM resulted in a $12.1 \%$ increase in strength $(p \leq 0.001)$. Among female, fat mass was marginally negatively associated with strength $(p=0.054)$ and LM, as well as among male, was positively associated with changes in strength in the adjusted model $(p=0.005)$. There was a $6.9 \%$ increase 


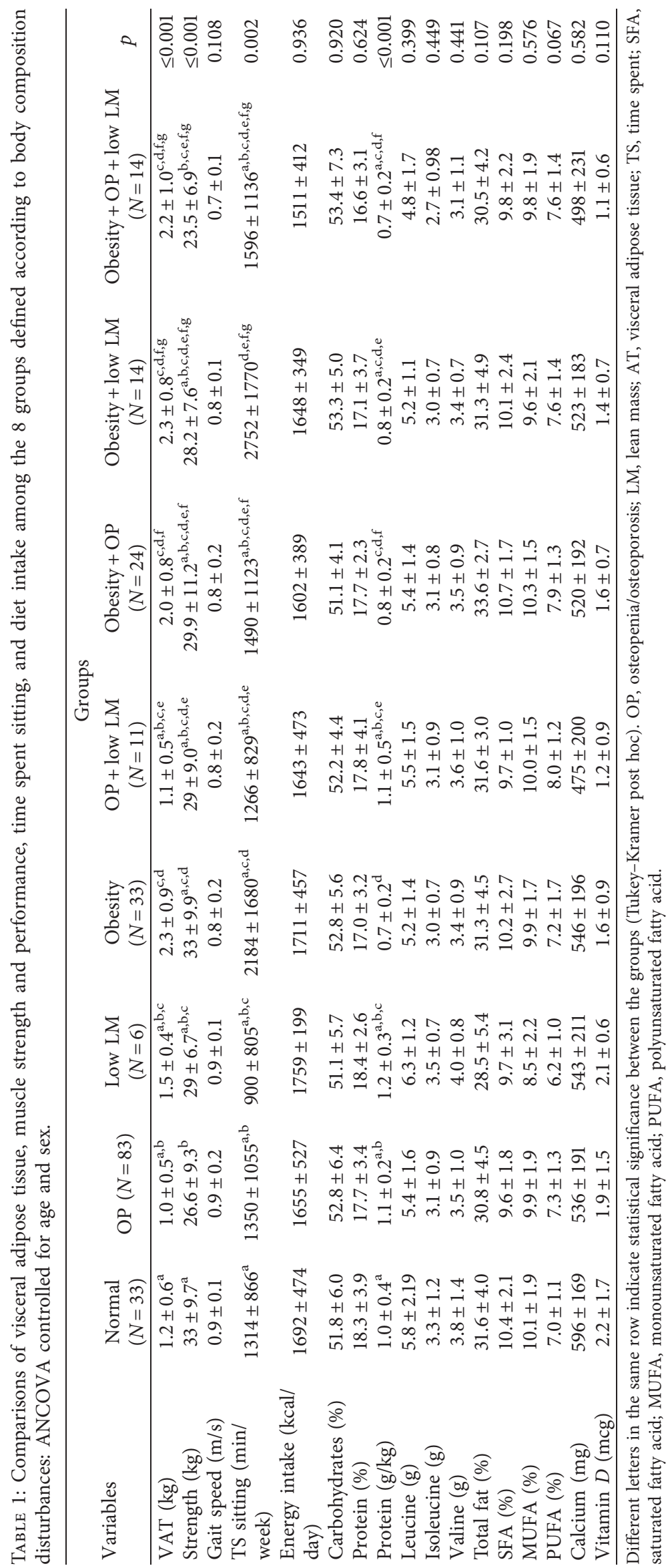


TABle 2: Pearson' chi-squared test performed to investigate the relationship between the 4 groups and socioeconomic and lifestyle variables.

\begin{tabular}{|c|c|c|c|c|c|c|}
\hline \multirow{2}{*}{ Variables } & \multicolumn{4}{|c|}{ Groups } & \multirow{2}{*}{$\chi^{2}$} & \multirow{2}{*}{$p$} \\
\hline & Normal $(N=33)$ & Low ALM and/or osteopenia $(N=100)$ & Obesity exclusive $(N=33)$ & Obesity + osteopenia and/or low ALM $(N=52)$ & & \\
\hline \multicolumn{7}{|l|}{ Sex } \\
\hline Female & $13(11.4)$ & $62(54.3)^{*}$ & $14(12.2)$ & $25(21.9)$ & \multirow{2}{*}{7.63} & \multirow{2}{*}{0.054} \\
\hline Male & $20(19.2)$ & $38(36.5)^{* *}$ & $19(18.2)$ & $27(25.9)$ & & \\
\hline \multicolumn{7}{|l|}{ Smoke } \\
\hline Yes & $9(27.27$ & $44(44.4 \%)$ & $13(39.3 \%)$ & $19(36.5 \%)$ & \multirow{2}{*}{3.26} & \multirow{2}{*}{0.352} \\
\hline No & $24(72.7 \%)$ & $55(55.5 \%)$ & $20(60.6 \%)$ & $33(63.4 \%)$ & & \\
\hline \multicolumn{7}{|l|}{ Alcohol consumer } \\
\hline Yes & $8(15.0 \%)$ & $22(41.5 \%)$ & $10(18.8 \%)$ & $13(24.5 \%)$ & \multirow[b]{2}{*}{0.88} & \multirow[b]{2}{*}{0.828} \\
\hline No & $25(15.2 \%)$ & $77(46.9 \%)$ & $23(14.0 \%)$ & $39(23.7 \%)$ & & \\
\hline \multicolumn{7}{|l|}{ Educational level } \\
\hline$\leq 9$ years & $17(11 \%)$ & $60(52 \%)$ & $14(12 \%)$ & $24(21 \%)$ & \multirow{3}{*}{12.17} & \multirow{3}{*}{0.058} \\
\hline $10-12$ years & $10(20 \%)$ & $23(46 \%)$ & $5(10 \%)$ & $12(24 \%)$ & & \\
\hline$\geq 13$ years & $6(12 \%)$ & $16(31 \%)$ & $14(28 \%)$ & $15(29 \%)$ & & \\
\hline \multicolumn{7}{|l|}{ Marital status } \\
\hline Married & $14(15 \%)$ & $46(49 \%)$ & $13(14 \%)$ & $20(22 \%)$ & \multirow{2}{*}{0.96} & \multirow{2}{*}{0.810} \\
\hline Not married & $19(15 \%)$ & $53(43 \%)$ & $20(16 \%)$ & $31(25 \%)$ & & \\
\hline \multicolumn{7}{|l|}{ Race } \\
\hline White & $14(10 \%)$ & $64(48 \%)$ & $21(16 \%)$ & $35(26 \%)$ & \multirow{2}{*}{6.62} & \multirow{2}{*}{0.084} \\
\hline Nonwhite & $19(23 \%)$ & $35(43 \%)$ & $12(15 \%)$ & $16(19 \%)$ & & \\
\hline \multicolumn{7}{|c|}{ Income (US\$/month)\# } \\
\hline$\leq 217.32$ & $13(19 \%)$ & $36(52 \%)$ & $7(10 \%)$ & $13(19 \%)$ & \multirow{3}{*}{14.9} & \multirow{3}{*}{0.021} \\
\hline$>217.32-651.96$ & $9(12 \%)$ & $28(37 \%)$ & $12(16 \%)$ & $26(35 \%)^{*}$ & & \\
\hline$>651.96$ & $5(14 \%)$ & $14(40 \%)$ & $11(31 \%)^{*}$ & $5(14 \%)$ & & \\
\hline \multicolumn{7}{|l|}{ Medications } \\
\hline 0 & $10(19 \%)$ & $23(42 \%)$ & $11(20 \%)$ & $10(19 \%)$ & \multirow{3}{*}{7.89} & \multirow{3}{*}{0.246} \\
\hline $1-4$ & $17(14 \%)$ & $61(52 \%)$ & $14(12 \%)$ & $26(22 \%)$ & & \\
\hline$\geq 5$ & $6(13 \%)$ & $16(35 \%)$ & $8(17 \%)$ & $16(35 \%)$ & & \\
\hline \multicolumn{7}{|l|}{ Leisure-time PA } \\
\hline$<150 \mathrm{~min} /$ week & $24(14 \%)$ & $76(44 \%)$ & $25(15 \%)$ & $47(27 \%)$ & \multirow{2}{*}{5.39} & 0 \\
\hline$\geq 150 \mathrm{~min} /$ week & $9(20 \%)$ & $23(51 \%)$ & $8(18 \%)$ & $5(11 \%)$ & & 0.145 \\
\hline
\end{tabular}

\# Income per capita, 1 US $\$=3.33$ Brazilian reals in $2015 .{ }^{*}$ Positive association in the residual analysis. ${ }^{* *}$ Negative association in the residual analysis.

TABLE 3: Linear regression models for the prediction of body composition components on strength changes.

\begin{tabular}{|c|c|c|c|c|c|c|}
\hline & Model $1 \beta$ (95\% IC) & $p$ & Model $2 \beta$ (95\% IC) & $p$ & Model $3 * \beta(95 \%$ IC $)$ & $p$ \\
\hline \multicolumn{7}{|l|}{ Men } \\
\hline LM (kg) & $1.05(0.71-1.39)$ & $\leq 0.001$ & $1.45(1.01-1.89)$ & $\leq 0.001$ & $1.21(0.75-1.67)$ & $\leq 0.001$ \\
\hline Total BF (kg) & $0.11(-0.05-0.29)$ & 0.183 & $-0.26(-0.46-0.06)$ & 0.009 & $-0.15(-0.38-0.07)$ & 0.186 \\
\hline FN BMD $(\mathrm{g} / \mathrm{cm} 2)$ & $3.98(-7.46-15.44)$ & 0.491 & $-0.61(-11.61-10.38)$ & 0.912 & $-6.87(-18.61-4.85)$ & 0.247 \\
\hline LS BMD (g/cm2) & $-4.15(-11.91-3.60)$ & 0.290 & $-3.55(-11.12-4.01)$ & 0.354 & $-0.36(-8.28-7.54)$ & 0.926 \\
\hline \multicolumn{7}{|l|}{ Women } \\
\hline LM $(\mathrm{kg})$ & $0.34(0.05-0.63)$ & 0.019 & $0.78(0.31-1.24)$ & 0.001 & $0.69(0.21-0.16)$ & 0.005 \\
\hline Total BF (kg) & $0.01(-0.08-0.10)$ & 0.824 & $-0.19(-0.33-0.04)$ & 0.011 & $-0.15(-0.31-0.00)$ & 0.054 \\
\hline FN BMD $(\mathrm{g} / \mathrm{cm} 2)$ & $4.92(-2.24-12.09)$ & 0.176 & $4.09(-4.65-12.84)$ & 0.356 & $0.79(-8.82-10.41)$ & 0.871 \\
\hline LS BMD (g/cm2) & $1.82(-3.02-6.67)$ & 0.458 & $-1.15(-7.08-4.77)$ & 0.700 & $0.47(-5.57-6.52)$ & 0.876 \\
\hline
\end{tabular}

LM, lean mass; BF, body fat; FN BMD, femoral neck bone mineral density; LS BMD, lumbar spine bone mineral density. Model 1, simple model for each body composition variable and strength. Model 2 , all body composition variables included in the same model. Model 3, model $2+$ protein intake $(\mathrm{g} / \mathrm{kg})+\mathrm{time}$ spent sitting (min/week) + age (years). ${ }^{*}$ For men: $F(7,93)=9.41$; adjusted $R^{2}=0.37(p \leq 0.001) .{ }^{*}$ For women: $F(7,102)=3.08$; adjusted $R^{2}=0.11(p=0.005)$.

and a $1.5 \%$ decrease in strength for each $10 \%$ increase in LM and in fat mass, respectively.

3.2. Discussion. In this study of 218 individuals, aged 63 (59-69) years, 52 (23\%) presented obesity associated with another body composition change, 14 (6\%) of which had the combination of the 3 conditions (obesity + OP + low LM).
Although an excessive adiposity state by itself has been associated with higher health risk behavior, its combination with reduced lean and bone mass led to an additional association with lower grip strength (confirming part of the hypothesis). The results allow us to hypothesize that people with obesity who show a high level of sedentary behavior and low protein intake may "evolve" to attain a state of simultaneous body composition changes, increasing the chance of 
exhibiting worse grip strength and the consequent establishment of a vicious cycle.

The prevalence of obesity $+\mathrm{OP}+$ low $\mathrm{LM}$ in this population of community-dwelling persons from 50 years was lower than most of previous investigations with osteosarcopenic obesity [14, 25-32], in which it ranged between $10 \%[29,30]$ and $31 \%$ [32]. Only Mo et al. (2018) [33] referred to a similar prevalence among Chinese older females (7\%) and Sasaki et al. (2020) [34] showed an even lower prevalence $(0.9 \%$ in older persons from the community and $4.4 \%$ in CVD patients). Most of the studies investigating osteosarcopenic obesity were conducted with Asian populations [14, 25-27, 30, 30, 32-34].

In addition to ethnic differences, comparing the prevalence of concomitant disturbances of body composition between studies is hampered by the lack of homogeneity in the criteria for diagnosing high fat mass and, especially, low LM [35]. Still, it is important to highlight that despite applying the terminology osteosarcopenic obesity, most studies that investigated this syndrome did not adopt current criteria for sarcopenia, since they considered only quantitative aspects of LM for diagnosis [14, 25-27, 30-33, 36].

In this study, we were careful to not use the term "sarcopenia" because we considered changes in LM only, given the low prevalence of people who showed strength lower than the suggested cutoffs. However, a dissociated evaluation of these parameters seems to emerge as a better approach. Alexandre et al. (2019) [37] showed that, although there are common predictors of reduced LM and strength (such as age and malnutrition), other variables have a different impact, depending on the outcome.

The main "impact" of the combination of obesity, low $\mathrm{LM}$, and OP seemed to be on functional status. As we did in our study, Ilichi et al. (2015) [31] and Szlejf et al. (2017) [28] observed lower grip strength among obese females with concomitant reductions in LM and BMD. The combination of these 3 conditions also resulted in slower walking speed, poorer balance [31], and 2 times increased tendency toward fragility and lower physical test battery score [28]. Similarly, Huo et al. (2016) [38] determined that sarcopenic obese individuals had lower BMD, grip strength, slower gait velocity, and poorer balance. In the present study, the linear models indicated that only LM was associated with strength, with no impact of BMD and not even of the fat mass (only marginal among women). In fact, Chain et al. (2020) [39] have demonstrated among a sample of Brazilian adults (33-81 years old) that dynapenia (age-related reduction in muscle strength) alone was not associated with BMD. The authors also observed that in dynapenic women, a higher fat mass was actually protective for the reduced BMD. In our study, fat mass was inversely associated with strength before undergoing adjustments that included age control. A review conducted by Tomlinson et al. (2016) [40] suggested that age classification may impact the relationship between fat mass and strength, since older obese have showed significantly lower knee extension isokinetic torque than their younger obese counterparts [41], requiring more research to assess what degree aging may impact on any association between obesity and muscle strength [40].
The impact of aging on body composition is undeniable. There is a redistribution of body fat, with increases in the abdominal and visceral area (VAT) and consequent release of inflammatory cytokines (e.g., TNF- $\alpha$, IL-1, IL-6, and CRP). Such inflammatory state has a detrimental impact on skeletal muscle by the ubiquitin-proteasome proteolytic pathway [42]. Additionally, with obesity, there is a reduction in the growth hormone $(\mathrm{GH})$ and an impairment of the insulin/IGF-1 pathway, anabolic hormones associated with protein synthesis, and muscle hypertrophy and regeneration [43]. Thus, the impact could be worse among older persons, since normal aging implies changes in optimal endocrine milieu [40] and involves the inflammaging phenomenon [44].

LM was the only body composition component with a significant linear relationship with strength among the participants of our study. Although it seems like an obvious result, Studenski et al. (2014) [19] in the FNIH Sarcopenia Project state that "it is clear that we can no longer assume that there is a direct and strong causal pathway from mass to strength," since there are many causes of weakness. However, a reduction in skeletal muscle tissue in aging is characterized by replacement of muscle fibers with noncontractile tissue, such as collagen and fat [45], an increase in fibrosis, changes in muscle metabolism, oxidative stress, and degeneration of the neuromuscular junction [46]. In addition, in older persons, the loss of muscle mass predominantly affects the type II (fast twitch) muscle fibers, which are associated with fiber type-specific decline in muscle stem cells content [47]. Considering such changes, it is expected for a progressive loss of muscle function over time. Losses in strength and performance increase the risk of independence loss and worsened quality of life. In addition, a vicious cycle can be triggered, in which the inability to perform daily activities worsens the primary condition.

The simultaneous changes in body composition have been demonstrated among adults aged from 50 years previously $[14,28,32,33]$. Such an early change may be a result of the association of the natural aging process with bad lifestyle habits. Recently, dos Santos and Gobbo (2020) [36] showed that older Brazilian women with obesity+low $\mathrm{LM}+$ osteopenia who remained insufficiently active throughout of the 24-month follow-up in leisure-time exercise and locomotion domains presented a higher risk for functional disability than those without such condition. The obesity and obesity + low LM groups spent more time sitting in a given week, suggesting higher levels of sedentary behavior. Sedentary behavior includes such activities as watching television or using computers, tablets, and mobiles, with minimum energy expenditure $(\leq 1.5 \mathrm{MET}$, metabolic equivalent of task) [48]. With these activities, there is not enough stimulus for protein synthesis [49] or bone turnover [50], and they also contribute to energy imbalance and a higher risk of obesity [51]. Additionally, the lower protein intake observed among all groups with obesity is another common predictor of reduced LM [49] and BMD [52]. Currently, $1.0 \mathrm{~g}-1.5 \mathrm{~g}$ of protein by bodyweight has been recommended for older persons aiming to maintain muscle and bone mass and to optimize functional status. 
A diet with higher protein intake and adequate calcium [53] can minimize an eventual increase in the excretion of this mineral. It is important to note that calcium is essential for muscle contraction [54] and bone mineralization [55]. Although there was no significant difference between the groups, calcium intake was inadequate among the entire sample, with a median of $535(382-655) \mathrm{mg}$. It is estimated that a minimum of $800-1,000 \mathrm{mg}$ of calcium is required daily for adults over 50 years to maintain homeostasis [24].

Limitations of the present study include the cross-sectional design, which does not allow for the establishment of any causal relationship. It was not possible to obtain the sample size estimated to provide a study power of $80 \%$ $\left(N_{\text {estimated }}=240\right.$ vs. $\left.N_{\text {obtained }}=218\right)$. In addition, the sample sizes of the groups were insufficiently homogenous, especially considering that the group characterized exclusively by low LM, limiting some statistical analyses. However, it is important to emphasize that the small number of people classified with low LM was not a consequence of the criteria used for diagnosis, as applying the European criteria did not change the prevalence (data not shown). Our findings are corroborated by Alexandre et al. (2019) [37] who also assessed residents of São Paulo (aged $69.8 \pm 0.6$ years) and observed a prevalence of low LM of 5\% when using the first edition of the European Working Group on Sarcopenia in Older People (EWGSOP) [56] criteria.

Including a sample of community-dwelling people, using a gold standard methodology for body composition assessment (DXA), strengthens the results. Additionally, in our sample, we included not only female but also male individuals who have not usually been considered in studies of osteosarcopenic obesity. Adults from 50 age also seem to be likely to show simultaneous changes in body composition, which reflects the impact of the lifestyle (especially, sedentary and protein intake habits). Considering the results of the present study, there is a need to conduct longitudinal investigations of the most common or natural direction of the disturbances in body composition, assessing the impact of aging and lifestyle together and apart. Standardizing the criteria to classify low LM (and sarcopenia) and even obesity (when defined according to body fat and not BMI) is indispensable. Moreover, given the public health context, it is also imperative to establish practical and less expensive criteria for screening the risk and diagnosing the changes in body composition.

\section{Conclusions}

The findings of this study confirm the physiological plausibility of concomitant obesity, reduced LM and OP in community-dwelling people. Although obesity itself was associated with higher level of sedentary behavior and lower protein intake, the combination of obesity with the other body composition changes presented the aggravating factor of being associated with lower grip strength. Thus, in a global context of demographic and nutritional transition, treating the simultaneous changes in body composition as an exclusive consequence of aging is very simplistic and can limit new and relevant findings regarding the potential physiological, social, and behavioral factors involved. Therefore, it is recommended that new studies be designed that aim to better understand the causes and impacts of simultaneous body composition changes, and to do so longitudinally.

\section{Data Availability}

All requests for data access should be addressed to the corresponding author. Proposals requesting data access will have to specify how they plan to use the data.

\section{Conflicts of Interest}

The authors declare that there are no conflicts of interest.

\section{Acknowledgments}

The authors would like to thank the São Paulo Research Foundation (FAPESP) and the National Council for Scientific and Technological Development (CNPq) (403393/2016-7) for financial support. Natasha A. G. de França has received a scholarship of São Paulo Research Foundation (FAPESP) (2014/26787-0) and (2015/07971-7) and Coordination of Superior Level Staff Improvement (CAPES).

\section{References}

[1] J. Z. Ilich, O. J. Kelly, J. E. Inglis, L. B. Panton, G. Duque, and M. J. Ormsbee, "Interrelationship among muscle, fat, and bone: connecting the dots on cellular, hormonal, and whole body levels," Ageing Research Reviews, vol. 15, pp. 51-60, 2014.

[2] M. Drey, C. C. Sieber, C. C. Sieber, T. Bertsch, J. M. Bauer, and R. Schmidmaier, "Osteosarcopenia is more than sarcopenia and osteopenia alone," Aging Clinical and Experimental Research, vol. 28, no. 5, pp. 895-899, 2016.

[3] K. Kobayashi, S. Imagama, K. Ando et al., "Epidemiology and effect on physical function of osteosarcopenia in communitydwelling elderly people in Japan," Modern Rheumatology, vol. 30, no. 3, pp. 592-597, 2020.

[4] F. Salech, C. Marquez, L. Lera, B. Angel, R. Saguez, and C. Albala, "Osteosarcopenia predicts falls, fractures, and mortality in Chilean community-dwelling older adults," Journal of the American Medical Directors Association, 2020.

[5] D. Scott, M. Seibel, R. Cumming et al., "Does combined osteopenia/osteoporosis and sarcopenia confer greater risk of falls and fracture than either condition alone in older men? The concord health and ageing in men project," The Journals of Gerontology: Series A, vol. 74, no. 6, pp. 827-834, 2019.

[6] B. Kirk, J. Zanker, and G. Duque, "Osteosarcopenia: epidemiology, diagnosis, and treatment-facts and numbers," Journal of Cachexia, Sarcopenia and Muscle, vol. 11, no. 3, pp. 609-618, 2020.

[7] H. P. Hirschfeld, R. Kinsella, and G. Duque, "Osteosarcopenia: where bone, muscle, and fat collide," Osteoporosis International, vol. 28, no. 10, pp. 2781-2790, 2017.

[8] R. Roubenoff, "Sarcopenic obesity: the confluence of two epidemics," Obesity Research, vol. 12, no. 6, pp. 887-888, 2004.

[9] W. Kim, S. G. Chung, K. Kim et al., “The relationship between body fat and bone mineral density in Korean men and women," Journal of Bone and Mineral Metabolism, vol. 32, no. 6, pp. 709-717, 2014. 
[10] M. J. Ormsbee, C. M. Prado, J. Z. Ilich et al., "Osteosarcopenic obesity: the role of bone, muscle, and fat on health," Journal of Cachexia, Sarcopenia and Muscle, vol. 5, no. 3, pp. 183-192, 2014.

[11] E. Atlantis, S. A. Martin, M. T. Haren, A. W. Taylor, G. A. Wittert, and S. Florey Adelaide Male Aging, "Lifestyle factors associated with age-related differences in body composition: the Florey Adelaide Male Aging Study," The American Journal of Clinical Nutrition, vol. 88, no. 1, pp. 95-104, 2008.

[12] U. T. Iwaniec and R. T. Turner, "Influence of body weight on bone mass, architecture and turnover," Journal of Endocrinology, vol. 230, no. 3, pp. R115-R130, 2016.

[13] G. Biolo, T. Cederholm, and M. Muscaritoli, "Muscle contractile and metabolic dysfunction is a common feature of sarcopenia of aging and chronic diseases: from sarcopenic obesity to cachexia," Clinical Nutrition, vol. 33, no. 5, pp. 737-748, 2014.

[14] J. Kim, Y. Lee, S. Kye, Y.-S. Chung, and O. Lee, "Association of serum vitamin D with osteosarcopenic obesity: korea national health and nutrition examination survey 2008-2010," Journal of Cachexia, Sarcopenia and Muscle, vol. 8, no. 2, pp. 259-266, 2017.

[15] A. I. Garcia-Gonzalez, Z. Axtle-Serrano, M. Lopez-Teros, C. Szlejf, A. Martinez-Ruiz, and O. Rosas-Carrasco, "Clinical interventions in osteosarcopenic obesity: nutrition, physical and psychological activity," Revista Médica del Instituto Mexicano del Seguro Social, vol. 56, no. Suppl 1, pp. S82-S93, 2018.

[16] M. C. G. P. Alves, M. M. L. Escuder, M. Goldbaum, M. B. D. A. Barros, R. M. Fisberg, and C. L. G. Cesar, "Sampling plan in health surveys, city of São Paulo, Brazil, 2015," Revista de Saúde Pública, vol. 52, p. 81, 2018.

[17] R. Fisberg, C. Sales, M. Fontanelli et al., "2015 health survey of São Paulo with Focus in nutrition: rationale, design, and procedures," Nutrients, vol. 10, no. 2, p. 169, 2018.

[18] F. Faul, E. Erdfelder, A.-G. Lang, and A. Buchner, "G * Power 3: a flexible statistical power analysis program for the social, behavioral, and biomedical sciences," Behavior Research Methods, vol. 39, no. 2, pp. 175-191, 2007.

[19] S. A. Studenski, K. W. Peters, D. E. Alley et al., “The FNIH sarcopenia project: rationale, study description, conference recommendations, and final estimates," The Journals of Gerontology: Series A, vol. 69, no. 5, pp. 547-558, 2014.

[20] T. L. Kelly, K. E. Wilson, and S. B. Heymsfield, "Dual energy $\mathrm{X}$-Ray absorptiometry body composition reference values from NHANES," PloS one, vol. 4, no. 9, Article ID e7038, 2009.

[21] J. A. Kanis, L. J. Melton, C. Christiansen, C. C. Johnston, and N. Khaltaev, "The diagnosis of osteoporosis," Journal of Bone and Mineral Research, vol. 9, no. 8, pp. 1137-1141, 1994.

[22] U. Harttig, J. Haubrock, J. Haubrock, S. Knüppel, and H. Boeing, "The MSM program: web-based statistics package for estimating usual dietary intake using the Multiple Source Method," European Journal of Clinical Nutrition, vol. 65, no. S1, pp. S87-S91, 2011.

[23] P. Trumbo, S. Schlicker, A. A. Yates, M. Poos, and Food Nutrition Board of the Institute of Medicine TNA, "Dietary reference intakes for energy, carbohydrate, fiber, fat, fatty acids, cholesterol, protein and amino acids," Journal of the American Dietetic Association, vol. 102, no. 11, pp. 1621-1630, 2002.
[24] A. C. Ross, "The 2011 report on dietary reference intakes for calcium and vitamin D," Public Health Nutrition, vol. 14, no. 5, pp. 938-939, 2011.

[25] J. Kim, Y. Lee, S. Kye et al., "Diet quality and osteosarcopenic obesity in community-dwelling adults 50 years and older," Maturitas, vol. 104, pp. 73-79, 2017.

[26] Y. M. Kim, S. Kim, Y. J. Won, and S. H. Kim, "Clinical manifestations and factors associated with osteosarcopenic obesity syndrome: a cross-sectional study in Koreans with obesity," Calcified Tissue International, vol. 105, no. 1, pp. 77-88, 2019.

[27] Y.-J. Bae, "Fruit intake and osteosarcopenic obesity in Korean postmenopausal women aged 50-64 years," Maturitas, vol. 134, pp. 41-46, 2020.

[28] C. Szlejf, L. Parra-Rodríguez, and O. Rosas-Carrasco, "Osteosarcopenic obesity: prevalence and relation with frailty and physical performance in middle-aged and older women," Journal of the American Medical Directors Association, vol. 18, no. 8, pp. e1-733, 2017.

[29] E. N. Kolbasi and F. Demirdag, "Prevalence of osteosarcopenic obesity in community-dwelling older adults: a crosssectional retrospective study," Arch Osteoporos, vol. 15, no. 1, p. 166, 2020.

[30] Y. Ma, W. Zhang, P. Han, M. Kohzuki, and Q. Guo, "Osteosarcopenic obesity associated with poor physical performance in the elderly Chinese community," Clinical Interventions in Aging, vol. 15, pp. 1343-1352, 2020.

[31] J. Z. Ilich, J. E. Inglis, O. J. Kelly, and D. L. McGee, "Osteosarcopenic obesity is associated with reduced handgrip strength, walking abilities, and balance in postmenopausal women," Osteoporosis International, vol. 26, no. 11, pp. 2587-2595, 2015.

[32] S. Park, W. Na, and C. Sohn, "Relationship between osteosarcopenic obesity and dietary inflammatory index in postmenopausal Korean women: 2009 to 2011 Korea National Health and Nutrition Examination Surveys," Journal of Clinical Biochemistry and Nutrition, vol. 63, no. 3, pp. 211216, 2018.

[33] D. Mo, P. Hsieh, H. Yu et al., "Osteosarcopenic obesity and its relationship with dyslipidemia in women from different ethnic groups of China," Archives of Osteoporosis, vol. 13, no. 1, p. $65,2018$.

[34] K.-i. Sasaki, T. Kakuma, M. Sasaki et al., "The prevalence of sarcopenia and subtypes in cardiovascular diseases, and a new diagnostic approach," Journal of Cardiology, vol. 76, no. 3, pp. 266-272, 2020.

[35] J. M. Bauer, A. J. Cruz-Jentoft, R. A. Fielding et al., "Is there enough evidence for osteosarcopenic obesity as a distinct entity? a critical literature review," Calcified Tissue International, vol. 105, no. 2, pp. 109-124, 2019.

[36] V. R. Dos Santos and L. A. Gobbo, "Physical activity is associated with functional capacity of older women with osteosarcopenic obesity: 24-month prospective study," European Journal of Clinical Nutrition, vol. 74, no. 6, pp. 912919, 2020.

[37] T. D. S. Alexandre, Y. A. D. O. Duarte, J. L. F. Santos, and M. L. Lebrão, "Prevalência e fatores associados à sarcopenia, dinapenia e sarcodinapenia em idosos residentes no Município de São Paulo - estudo SABE," Revista Brasileira de Epidemiologia, vol. 21, no. suppl 2, Article ID e180009, 2019.

[38] Y. R. Huo, P. Suriyaarachchi, F. Gomez et al., "Phenotype of sarcopenic obesity in older individuals with a history of falling," Archives of Gerontology and Geriatrics, vol. 65, pp. 255-259, 2016. 
[39] A. Chain, E. Faerstein, V. Wahrlich, and F. F. Bezerra, "Obesity, dynapenia, and their combination: implications for bone mineral density in Brazilian adults-the Pró-Saúde study," Nutrition, vol. 81, p. 110898, 2021.

[40] D. J. Tomlinson, R. M. Erskine, C. I. Morse, K. Winwood, and G. Onambélé-Pearson, "The impact of obesity on skeletal muscle strength and structure through adolescence to old age," Biogerontology, vol. 17, no. 3, pp. 467-483, 2016.

[41] M. Hulens, G. Vansant, R. Lysens, A. Claessens, E. Muls, and S. Brumagne, "Study of differences in peripheral muscle strength of lean versus obese women: an allometric approach," International Journal of Obesity, vol. 25, no. 5, pp. 676-681, 2001.

[42] L. Larsson, H. Degens, M. Li et al., "Sarcopenia: aging-related loss of muscle mass and function," Physiological Reviews, vol. 99, no. 1, pp. 427-511, 2019.

[43] B. Roy, M. E. Curtis, L. S. Fears, S. N. Nahashon, and H. M. Fentress, "Molecular mechanisms of obesity-induced osteoporosis and muscle atrophy," Frontiers in Physiology, vol. 7, p. 439, 2016.

[44] C. Franceschi, P. Garagnani, P. Parini, C. Giuliani, and A. Santoro, "Inflammaging: a new immune-metabolic viewpoint for age-related diseases," Nature Reviews Endocrinology, vol. 14, no. 10, pp. 576-590, 2018.

[45] J. A. Kent-Braun, A. V. Ng, and K. Young, "Skeletal muscle contractile and noncontractile components in young and older women and men," Journal of Applied Physiology, vol. 88, no. 2, pp. 662-668, 2000.

[46] R. J. S. Dhillon and S. Hasni, "Pathogenesis and management of sarcopenia," Clinics in Geriatric Medicine, vol. 33, no. 1, pp. 17-26, 2017.

[47] L. B. Verdijk, R. Koopman, G. Schaart, K. Meijer, H. H. C. M. Savelberg, and L. J. C. van Loon, "Satellite cell content is specifically reduced in type II skeletal muscle fibers in the elderly," American Journal of Physiology-Endocrinology and Metabolism, vol. 292, no. 1, pp. E151-E157, 2007.

[48] N. Sedentary Behaviour Research, "Letter to the editor: standardized use of the terms sedentary and sedentary behaviours," Applied Physiology, Nutrition, and Metabolism, vol. 37, no. 3, pp. 540-542, 2012.

[49] D. R. Moore, T. A. Churchward-Venne, O. Witard et al., "Protein ingestion to stimulate myofibrillar protein synthesis requires greater relative protein intakes in healthy older versus younger men," The Journals of Gerontology Series A: Biological Sciences and Medical Sciences, vol. 70, no. 1, pp. 57-62, 2015.

[50] A. Neve, A. Corrado, and F. P. Cantatore, "Osteocytes: central conductors of bone biology in normal and pathological conditions," Acta Physiologica, vol. 204, no. 3, pp. 317-330, 2012.

[51] E. Archer, C. J. Lavie, and J. O. Hill, "The contributions of diet, genes, and physical activity to the etiology of obesity: contrary evidence and consilience," Progress in Cardiovascular Diseases, vol. 61, no. 2, pp. 89-102, 2018.

[52] J. M. Beasley, A. Z. LaCroix, J. C. Larson et al., "Biomarkercalibrated protein intake and bone health in the Women's Health Initiative clinical trials and observational study," The American Journal of Clinical Nutrition, vol. 99, no. 4, pp. 934-940, 2014.

[53] J. H. O’Keefe, N. Bergman, P. Carrera-Bastos, M. FontesVillalba, J. J. DiNicolantonio, and L. Cordain, "Nutritional strategies for skeletal and cardiovascular health: hard bones, soft arteries, rather than vice versa," Open Heart, vol. 3, no. 1, Article ID e000325, 2016.
[54] M. Brotto, "Aging, sarcopenia and store-operated calcium entry," Cell Cycle, vol. 10, no. 24, pp. 4201-4202, 2011.

[55] A. Hossein-nezhad and M. F. Holick, "Vitamin D for health: a global perspective," Mayo Clinic Proceedings, vol. 88, no. 7, pp. 720-755, 2013.

[56] A. J. Cruz-Jentoft, J. P. Baeyens, J. M. Bauer et al., "Sarcopenia: European consensus on definition and diagnosis: report of the European working group on sarcopenia in older people," Age and Ageing, vol. 39, no. 4, pp. 412-423, 2010. 